\title{
Linfadenitis tuberculosa en infección VIH. Hallazgos histológicos
}

\author{
Fernando Arévalo ${ }^{1}$, José Cabanillas ${ }^{2}$
}

\begin{abstract}
Resumen Introducción: La coinfección de virus de inmundeficiencia humana(VIH) y tuberculosis altera la presentación histológica de la tuberculosis; esto es particularmente frecuente en la linfadenitis tuberculosa. Objetivos: Identificar las diferencias histológicas más importantes entre linfadenitis tuberculosa asociada a VIH y no asociada a VIH. Diseño: Estudio descriptivo, transversal, comparativo. Lugar: Servicio de Anatomía Patológica, Hospital Dos de Mayo. Participantes: Pacientes con linfadenitis tuberculoso asociada o no a VIH. Intervenciones: Se realizó un estudio en todos los casos diagnosticados como linfadenitis tuberculosa asociados a VIH y no asociados a VIH, durante los años 2005 y primera mitad del 2006. Principales medidas de resultados: Presentación histológica de la tuberculosis. Resultados: Se identificó 22 casos de linfadenitis tuberculosa, de los cuales 27,3\% presentaba coinfección con VIH. Se identificó necrosis caseosa en 83,3\% de los casos asociados a VIHyen $100 \%$ de los no asociados a VIH $(p=0,00)$. La presencia de bacilos ácido alcohol resistentes se observó en $50 \%$ de los casos asociados a VIHy en ningún caso de los VIH negativos ( $p=0,01)$. Adicionalmente, se obtuvo un riesgo atribuible de 52,3\% para la presencia de células de Langhans y el desarrollo de necrosis caseosa. Conclusiones: Se ha encontrado mayor presencia de mycobacterium en los pacientes VIH positivos y mayor presencia de necrosis caseosa en los pacientes VIHnegativos.
\end{abstract}

Palabrasclave Tuberculosis ganglionar, HIV; necrosis; células de Langhans gigantes.

Tuberculous lymphadenitis in HIV infection. Histological findings

\begin{abstract}
Introduction: Coinfection of human virus immunodeficiency $(\mathrm{VIH})$ and tuberculosis changes the histological features of tuberculosis; this is particularly frequent in tuberculous lymphadenitis. Objective: To identify main histological diferences between tuberculous lymphadenitis associated to VIH and not associated to VIH. Design: Descriptive, transversal, comparative stufy. Setting: Pathological Anatomy Service, Dos de Mayo Hospital. Participants: Patients with tuberculous lymphadenitis associated or not to VIH. Interventions: Study of histological differences between lymphadenitis tuberculosis associated with HIV and non associated with HIV, during 2005 and first half of 2006. Main outcome measures: Histological presentation of tuberculosis. Results: Twenty-two cases of tuberculous lymphadenitis were identified, 27,3\% showed VIH coinfection; caseum necrosis was identified in $83,3 \%$ of cases HIV-associated and in $100 \%$ of nonHIV associated cases $(p=0,00)$. Koch bacillus was identified in

1 Medico residente de $3^{\circ}$ año. Anatomía patológica del Hospital Nacional Dos de Mayo. Lima, Perú.

2 Médico Anátomo-patólogo asistente del Hospital Nacional Dos de Mayo. Lima, Perú.
\end{abstract}

$50 \%$ of associated HIV cases and it was not identified in any non related HIV case $(p=0,01)$. We also found an attributable risk of $52,3 \%$ for the development of caseum necrosis and presence of Langhans cells. Conclusions: We found higher presence of mycobacterium in HIV positive patients and higher presence of caseum necrosis in HIVnegative patients.

Key words: Tuberculosis, lymphanode; HIV; necrosis; giant cells, Langhans.

\section{INTRODUCCIÓN}

La tuberculosis es considerada, hoy en día, una enfermedad emergente, debido en parte a que la coinfección con el virus de la inmunodeficiencia humana ha producido un incremento en su incidencia durante los últimos años $\left({ }^{1,2}\right)$, especialmente en países que se veían libres del mycobacterium. La asociación con VIH, además de elevar la morbilidad y las tasas de incidencia, también altera el curso clínico y la representación histológica de la tuberculosis, modifi- 
cando su presentación habitual. El compromiso ganglionar es una de las localizaciones extrapulmonares más frecuentes de la tuberculosis y es especialmente elevada en pacientes con infección por VIH $\left.{ }^{3,4}\right)$. Bekedam demostró que, la presencia de VIH en la linfadenitis tuberculosa disminuye el poder diagnóstico tanto del cultivo como de la histología de tuberculosis $\left({ }^{1}\right)$. Los criterios histológicos usados para diagnosticar una linfadenitis granulomatosa como tuberculosa son la presencia de necrosis caseosa y la reacción granulomatosa, con presencia de células de tipo Langhans. Sin embargo, en un paciente inmunosuprimido, el patrón histológico puede variar, por la disminución de linfocitos CD4; y, por consiguiente, se presentará una respuesta inmunológica alterada. Nos propusimos determinar las características histológicas de la linfadenitis tuberculosa en pacientes con diagnóstico de infección por VIH y compararlos con un grupo de casos con diagnóstico de linfadenitis tuberculosa sin VIH, con el objeto de aportar mayor conocimiento del comportamiento de ambas patologías infecciosas, frecuentes en nuestro país.

\section{MATERIALES Y MÉTODOS}

El presente trabajo es descriptivo retrospectivo comparativo. Se estudió todos los casos diagnosticados como linfadenitis tuberculosa en el servicio de Anatomía Patológica del Hospital Dos de Mayo (HN2M), durante los años 2005 y primera mitad del 2006, en pacientes diagnosticados como linfadenitis tuberculosa, resultando 12 casos con un total de 42 casos de ganglios informados como procesos reactivos no neoplásicos. Se definió como caso de linfadenitis tuberculosa aquel que en los cortes microscópicos presentara al menos uno de los 2 criterios siguientes: a) reacción granulomatosa completa, b) necrosis caseosa con reacción granulomatosa incompleta, c) presencia de bacilos ácido alcohol resistentes con la coloración de Ziehl Nielsen. No se realizó cultivos microbiológicos, dado que no contábamos con muestra fresca para tal fin.

Se incluyó las siguientes variables: edad, sexo, VIH, células gigantes tipo Langhans, células epitelioides, necrosis caseosa, bacilo de Koch (BK). La variable VIH fue categorizada como presencia o ausencia de la infección, según lo consignado en la historia clínica del paciente. El resto de variables fue categorizado como ausentes o presentes.

La información clínica fue extraída del banco de datos del sistema del servicio de Anatomía Patológica y archivo de historias clínicas del HN2M. Los cortes histológicos fueron revisados, a fin de precisar las variables histológicas descritas. Los resultados fueron presentados en porcentajes y la significancia estadística fue analizada mediante la prueba t student para muestras independientes, para un nivel de confianza de 0,05, con ayuda del programa SPSS 13,0.

\section{RESULTADOS}

Se identificó 22 casos de linfadenitis tuberculosa, de los cuales $27,3 \%$ presentaba coinfección VIH, siendo 12 casos de género femenino y 10 de género masculino. La edad promedio de los pacientes con linfadenitis tuberculosa y VIH fue 38 años, en comparación con la edad promedio de los casos no asociados a VIH, que fue 37,5 años; la mayoría de los pacientes atendidos en el hospital son adultos.

La necrosis caseosa estuvo presente en $83,3 \%$ de los casos con VIH y en el $100 \%$ de los casos no asociados a VIH. La coloración Ziehl Nielsen mostró bacilos ácido alcohol resistentes en $50 \%$ de los casos con VIH y en ningún caso de los no asociados a VIH. Las células de Langhans fueron identificadas en $75 \%$ de casos no asociados a 
VIH y en $66,6 \%$ de los casos asociados a VIH. La diferencia fue estadísticamente significativa en las variables BK y en la necrosis caseosa, para un nivel de significancia de 0,05 (Tabla 1).

Tabla 1.-Características de linfadenitis tuberculosa, según presencia de VIH.

\begin{tabular}{lrrrrrr}
\hline & \multicolumn{2}{c}{ VIH } & & \multicolumn{2}{c}{ No VIH } & \\
\cline { 2 - 3 } & $\mathrm{n}$ & $\%$ & & $\mathrm{n}$ & $\%$ & \multicolumn{1}{c}{$p$} \\
\hline Necrosis caseosa & 5 & 83,3 & & 16 & 100 & 0,00 \\
BK + & 3 & 50 & & 0 & & 0,001 \\
Células Langhans & 4 & 66,6 & & 12 & 75 & 0,5 \\
Células epitelioides & 6 & 100 & & 16 & 100 & \\
Total & 6 & 100 & & 16 & 100 &
\end{tabular}

Se relacionó las variables células de Langhans y necrosis caseosa, a fin de medir la importancia de la presencia de células de Langhans como factor asociado al desarrollo de necrosis caseosa; obtuvimos un riesgo atribuible de 52,3\% (Tabla 2).

Tabla 2. Relación entre células de Langhans y necrosis caseosa.

\begin{tabular}{lrrrrrr}
\hline & \multicolumn{2}{c}{ Necrosis caseosa } & & \multicolumn{2}{c}{ No necrosis caseosa } \\
\cline { 2 - 3 } \cline { 5 - 6 } & $\mathrm{n}$ & $\%$ & & $\mathrm{n}$ & $\%$ \\
\hline Langhans & 16 & 76,1 & & 0 & 0 \\
No Langhans & 5 & 23,8 & & 1 & 100 \\
Total & 21 & 100 & & 1 & 100 \\
\hline
\end{tabular}

\section{DISCUSIÓN}

Rajasakaran y col. hallaron $18,8 \%$ de casos VIH positivos en 85 pacientes diagnosticados como linfadenitis tuberculosa, en su trabajado realizado en la India $\left(^{5}\right)$, mientras que $28,1 \%$ de 1340 pacientes diagnosticados como linfadenitis tuberculosa fueron VIH positivos en el trabajo de Chuquiyauri y col. $\left(^{6}\right)$ realizado en el Hospital Cayetano Heredia. Nosotros obtuvimos un porcentaje similar al de Chuquiyauri, de $27,3 \%$. Creemos que la coinfección de VIH y tuberculosis es el resultado de la presencia, en nuestra población, de factores de riesgo comu- nes a ambas enfermedades, tales como la desnutrición y la falta de educación. El promedio de edad de los pacientes con linfadenitis tuberculosa con VIH fue 38,16 años, mientras que el promedio de edad de los pacientes con linfadenitis tuberculosa sin VIH fue 37. Según el reporte global de tuberculosis, de la WHO en las Américas $\left({ }^{7}\right)$, la mayor parte de los casos corresponde a adultos jóvenes, es decir en la etapa más productiva de la vida. La mayor exposición y contacto social serían las razones que explican la mayor frecuencia de tuberculosis VIH en este grupo generacional. La OMS ha informado de niveles extraordinarios de infección y muerte en mujeres y niñas, con más de 900 millones infectadas actualmente por el bacilo en todas partes del mundo. En nuestro trabajo, 12 casos fueron femeninos y 10 masculinos. No obstante, el género es una variable un tanto sesgada en el Hospital Nacional Dos De Mayo, el cual atiende una población tradicionalmente adulta masculina.

La necrosis caseosa es un fenómeno constante de la tuberculosis activa. Microscópicamente, se caracteriza por ser un material amorfo, eosinofílico, con algunas partículas incluidas, las que corresponderían a restos celulares y depósitos minerales. Este tipo de necrosis es inusual para otras infecciones con respuesta granulomatosa, debido a que en aquellas la necrosis se instala mucho antes que la respuesta granulomatosa. Nosotros observamos presencia de necrosis caseosa en cuadros histológicos con y sin células de Langhans. Al realizar una tabla de contingencia entre las variables necrosis caseosa y la presencia de células de Langhans, obtuvimos un riesgo atribuible de $52,3 \%$ para el desarrollo de necrosis caseosa en presencia de células de Langhans. Este hallazgo nos permite suponer que el desarrollo de necrosis caseosa depende de la presencia del macrófago activado, es decir de la célula de Langhans; pero, también participarían otros factores. Algunos autores describen que, en la pared 
del mycobacterium se encuentra un glicolípido tóxico llamado factor cordonal, el cual tendría una participación directa en el desarrollo de la necrosis inicial de la tuberculosis $\left(^{8}\right)$. Un curioso fenómeno observado en 2 casos de pacientes con VIH fue la disposición de la necrosis caseosa en forma angulada; no hemos encontrado bibliografía que mencione este hecho particular.

Se admite que la activación del macrófago es la clave para la formación del granuloma y la destrucción de la micobacteria. Esta activación depende de la participación de citoquinas, tales como interferón- $\gamma$ e IL-4, las que permiten el desarrollo de células epitelioides y su fusión, para formar las células gigantes tipo Langhans $\left({ }^{9,10}\right)$. La presencia de células de Langhans fue mayor en los casos no asociados a VIH. Creemos que la diferencia se explica por la disminución de linfocitos $\mathrm{T}$, que sintetizan tanto IL4 e interferón. No obstante, no se observó lo mismo con la presencia de células

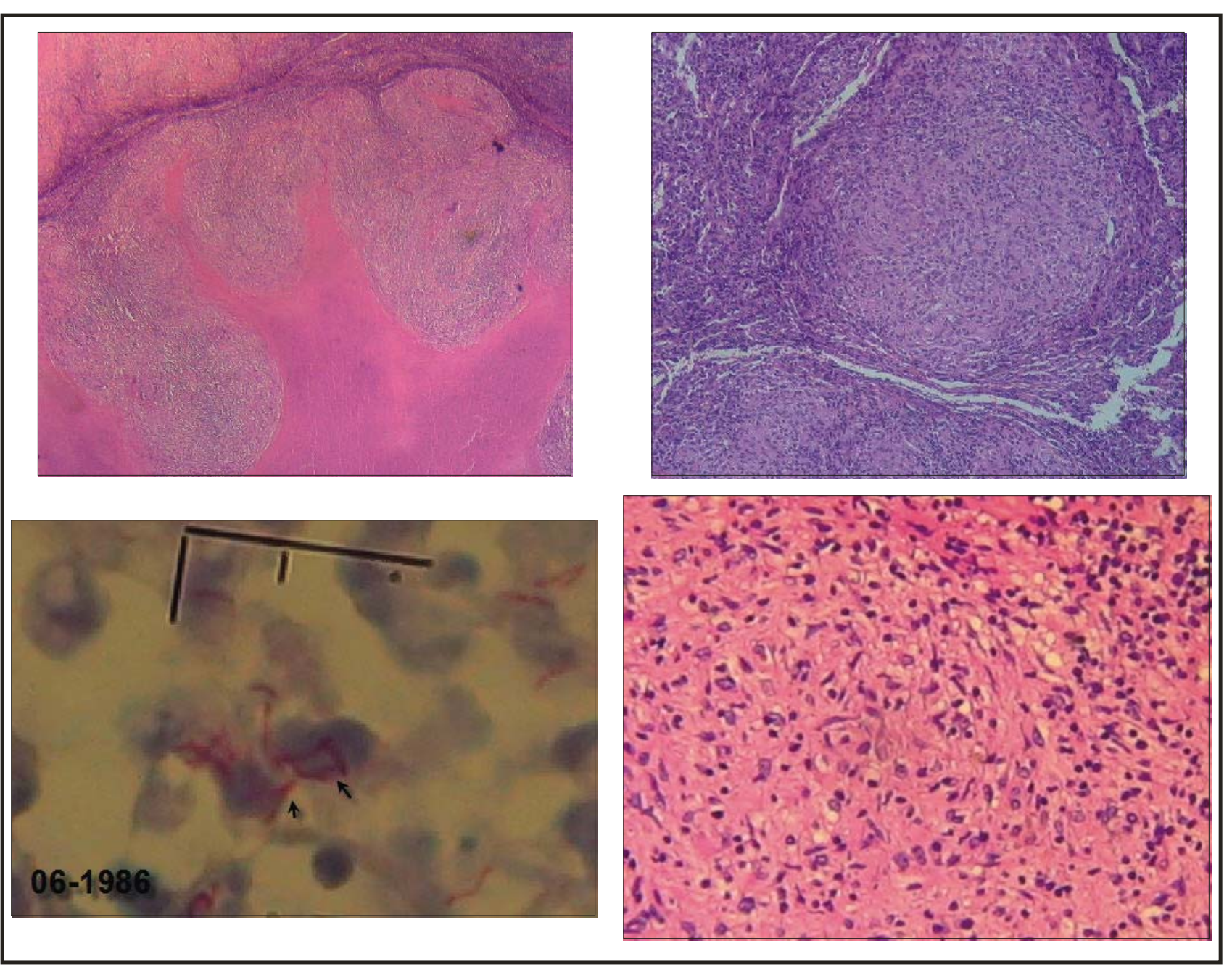

Figura 1. La fotografía superior izquierda muestra la presencia de necrosis caseosa en un ganglio de un paciente con VIH.

Nótese la curiosa distribución de la necrosis en forma angulada(HE 2000X). La fotografía superior derecha muestra la presencia de granulomas incompletos en un ganglio de un paciente VIH positivo; estos granulomas están constituidos solo por células epitelioides (HE 200X)s. La fotografía inferior izquierda señala la presencia de mycobacterium tuberculosis con aceite de inmersión.

La fotografía inferior derecha muestra células epitelioides a mayor aumento, en un ganglio de un paciente VIH positivo (400x). 
epitelioides, las que estuvieron presentes en todos los casos con infección y sin infección VIH.

La identificación del mycobacterium es fácil en los estadios iniciales de la infección. Pero, cuando la respuesta de hipersensibilidad se instala completamente, los bacilos son escasos y difíciles de encontrar por muchas razones: hipoxia, pH ácido, y la misma respuesta de hipersensibilidad $\left({ }^{11}\right)$ (Figura 1). Cuando se les logra identificar, los bacilos son grosella, arrosariados, delgados, curvados y usualmente miden entre 1 y 5 micras $\left({ }^{12}\right)$. No hemos identificado bacilos ácido alcohol resistentes en los casos libres de infección VIH, mientras que el $50 \%$ de los casos asociados a VIH fueron BK positivos. La mayor frecuencia de bacilos ácido alcohol resistentes se explica con la deficiencia de la inmunidad celular presente en un contexto de VIH. Resaltamos el hecho que las mycobacterium identificadas fueron más grandes del promedio usual. En conclusión, de todos los casos diagnosticados como linfadenitis tuberculosa, $27,3 \%$ presentaba coinfección con VIH. Las diferencias histológicas estadísticamente significativas fueron la necrosis caseosa, más frecuente en los casos VIH negativos, y la presencia de bacilos ácido alcohol resistentes, más frecuente en los casos VIH positivos. Estas diferencias histológicas pueden influenciar en el pronóstico de la TBC y TBC asociada a VIH.

\section{REFERENCIAS BIBLIOGRÁFICAS}

1. Bekedam HJ, Boeree M, Kamenya A, Liomba G, Ngwira B, Subramanyam VR, et al, Tuberculous lymphadenitis, a diagnostic problem in areas of high prevalence of HIV and tuberculosis. Trans R Soc Trop Med Hyg. 1997;91(3):2947.

2. Barnes PF, Bloch AB, Davidson PT. Tuberculosis in patients with human immunodeficiency virus infection. N Engl J Med. 1991;324:1644-50.
3. Raviglione $\mathrm{MC}, \mathrm{O}^{\prime}$ Brien RJ. Tuberculosis. Principios de Medicina Interna, Harrison. $14^{\text {a }}$ edición. Madrid: McGrawHill Interamericana; 1998. p. 1149-61.

4. Connor DH, Chandler FW, Swartz DA, Manz HJ, Lack EF. Pathology of Infectious disease. $1^{\text {st }}$ ed. London: Ed Appleton \& Lange; 1997.

5. Rajasekaran S, Gunasekaran M, Jayakumar D. Tuberculous cervical lymphadenitis in HIV positive and negative patients. Ind J tub. 2001;48:201.

6. Chuquiyauri HR, Verdonck BK, González L. Morbimortalidad de pacientes con tuberculosis hospitalizados en el Departamento de enfermedades infecciosas, tropicales y dermatológicas del Hospital Nacional Cayetano Heredia, Lima - Perú, entre los años 1990 y 2000. Rev Med Hered. 2004;15(4):203.

7. Organización Mundial de la Salud (OMS). Global Tuberculosis Report. Datos correspondientes al año 2003. Ginebra: OMS; 2005.

8. Silva CL, Ekizlerian SM, Fazioli RA. Role of cord factor in the modulation of infection caused by mycobacteria. Am J Pathology. 1985;118:238-47.

9. Muller H, Takeshita M. In situ immunophenotype of macrophages and lymphocytes in granuloma formation of tuberculous lymphadenitis in HIV-infected and immunocompctent patients. Res Virol. 1991;142:159.

10. Hayase Y, Tobika K. Cervical tuberculous lymphadenitis in a frequent traveler to endemic areas of tuberculosis. Intern Med. 1997;36(3):211-3.

11. Hopewell PC. Overview of clinical tuberculosis. En: Bloom BR, ed. Tuberculosis: pathogenesis, protection, and control. Washington, DC: American Society for Microbiology; 1994. p. 25-46.

12. Wear DJ, Hadfield TL, Connor DH, Neafie RC, Banks IS, Meyers WM, et al. Periodic acid-Shiff reaction stains. Mycobacterium tuberculosis, Mycobacterium leprae, Mycobacterium ulcerans, Mycobacterium Chelonae (abscessus) and Mycobacterium kansassi. Arch Pathol Lab Med. 1985;109:701-2.

Manuscrito recibido el 01 de setiembre de 2006 y aceptado para publicación el 29 setiembre de 2006.

\section{Correspondencia:}

Fernando Antonio Arévalo

Anatomía Patológica. Hospital Nacional Dos de Mayo

Av. Grau cuadra 13. Parque Historia de la Medicina.

Lima 1, Perú.

Correo-e:fer13013@hotmail.com 